\title{
Biomechanical and Refractive Results of Transepithelial Cross-linking Treatment in Keratoconic Eyes
}

\author{
Aylin Kılıç, Cynthia J Roberts
}

\begin{abstract}
Purpose: To analyze change in visual acuity (VA), refractive outcomes, corneal compensated intraocular pressure (IOPCC), corneal hysteresis $(\mathrm{CH})$ and cornea resistance factor $(\mathrm{CRF})$ after transepithelial cross-linking $(\mathrm{CXL})$ treatment.
\end{abstract}

Setting: Kudret Eye Hospital, Ankara, Turkey

Materials and methods: A total of 32 eyes of 22 patients diagnosed with keratoconus were included in this retrospective study. Changes in VA, spheric and cylinderic refraction for all eyes were analyzed before and 3 months after transepithelial CXL. In addition, a subset of 14 eyes had data acquired using the ocular response analyzer (ORA), and for these eyes, IO PCC, $\mathrm{CH}$ and $\mathrm{CRF}$ were recorded as well as a measurement of the amplitude of the first peak in the infrared signal (peak 1), used to identify the inward applanation event. The ORA parameters were also compared before and 3 months after treatment.

Results: Uncorrected and best corrected VA increased 1.76 and $1.61 \mathrm{~S}$ nellen lines $(p<0.05)$; spheric and cylinderic refractions improved 0.74 and $0.43 D(p>0.05)$ respectively. Mean changes in IOP CC, CRF and $\mathrm{CH}$ were not significantly different $(p>0.05)$. However, mean peak 1 signal value increased significantly $(p<0.05)$.

Conclusion: Transepithelial CXL is effective in VA improvement in the short-term with an increase in the peak 1 signal value which is consistent with an increase in stiffness of cornea, whereas IOPCC, CRF and $\mathrm{CH}$ values remained the same.

Keywords: Keratoconus, Cross-linking, Hysterestis, Transepithelial, Cornea biomechanical.

How to cite this article: Kilıç A, Roberts CJ. Biomechanical and Refractive Results of Transepithelial Cross-linking Treatment in Keratoconic Eyes. Int J Kerat Ect Cor Dis 2012;1(2):75-78.

\section{Source of support: Nil}

\section{Conflict of interest: None}

\section{INTRODUCTION}

The biomechanical properties of the cornea are primarily determined by the collagen fibers within the storma and degree of interfibrillar linkage. ${ }^{1}$ Collagen cross-linking (CXL) in the cornea using ultraviolet A (UVA) light and the photosensitizer riboflavin has been developed recently as a novel method leading to a significant increase in the mechanical stiffness of the cornea, as demonstrated in biomechanical stress-strain measurements. ${ }^{2,3}$

Keratoconus starts at puberty, progressing in approximately $20 \%$ to such an extent that penetrating keratoplasty becomes necessary. ${ }^{4,5} \mathrm{~W}$ ollensak et al ${ }^{6}$ reported that corneal collagen CXL with UVA and riboflavin leads to a mean of 2.00 diopters (D) of topographic flattening as an alternative management of keratoconus.

Increase in biomechanical strength after $\mathrm{CX} L$ has been demonstrated by stress-strain measurements, as well as thermal and enzyme digestion studies. Stress-strain studies have been performed in human donor corneas. The change in human donor corneas was a si gnificant increase in el astic modulus. ${ }^{13}$

In this study, changes in visual acuity (VA), refraction and specific ocular response analyzer (ORA) values after $C X L$ treatment were analyzed to evaluate visual and biomechancial response to a transepithelial procedure.

\section{MATERIALS AND METHODS}

In this retrospective study, 32 eyes of 22 patients (14 males and eight female) with keratoconus were included. Informed consent was obtained from all patients before the CXL procedure.

Uncorrected visual acuity (UCVA; Snellen line), best corrected visual acuity (BCVA; Snellen line), manifest spheric and cylinderic refraction measurements, and keratometric data were obtained before and 3 months following treatment of transepithelial $\mathrm{CXL}$.

On a subset of 14 eyes, measurements were obtained with the ORA, including cornal compensated intraocular pressure (IOPCC), corneal hysteresis $(\mathrm{CH})$ and corneal resistance factor (CRF). In addition, the amplitude of the first peak in the infrared signal (peak 1), corresponding to the first inward applanation event, was recorded. Statistical comparisons of preoperative and postoperative values were performed using the paired two samples test for UCVA, $B C V A$, pachymetry $(C C T)$, mean $K$ values, IOPCC, $C H$, CRF and peak 1 value.

\section{Surgical Technique}

All eyes underwent standardized $C X L$ procedure (supplies obtained from Pechke Company) consisting of a 30 -minute application of UVA light $\left(3.0 \mathrm{~mW} / \mathrm{cm}^{2}\right.$ at $370 \mathrm{~nm}$ ) to the central $7.0 \mathrm{~mm}$ of the cornea combined with topical application of riboflavin solution $(0.1 \%$ riboflavin5 -phosphate and dextran) every 3 minutes without removal of epithelium, pilokarpin hidroklorür $2 \%$ every 10 and proparacaine every 5 for 30 minutes and $20 \%$ alcohol 
application for 25 second before UVA application. The original protocol reported in the literature involved the removal of the central epithelium with a blunt spatula. However, we did not remove the epithelium and performed the technique described by Chan et al. ${ }^{7}$

After treatment artificial tear drop was recommended for 3 days.

\section{RESULTS}

The mean age of patients was $26.37 \pm 8.41$ years. M ean UCV A and BCV A significantly improved from $3.04 \pm 2.15$ and $4.98 \pm 2.48$ lines preoperative, to $4.80 \pm 2.37$ and 6.59 \pm 2.34 lines (Snellen acuity) postoperative, respectively $(p<0.05)$ (Table 1). The magnitude of improvements in UCVA and BCVA were $1.76 \pm 2.27$ lines and $1.61 \pm 2.52$ Snellen lines.

Preoperative mean spheric, cylinderic refraction and mean $K$ value did not show a significant change from $-3.21 \pm 3.13,-2.18 \pm 1.55$ and $47.44 \pm 4.95 D$ to $-2.47 \pm$ $2.51,-1.75 \pm 1.32$ and $46.33 \pm 3.62 \mathrm{D}$, respectively $(p>0.05)$ (Table 1$)$.

Preoperative IOPCC, CRF and $\mathrm{CH}$ were $16.58 \pm 4.94$, $6.67 \pm 1.88$ and $7.17 \pm 2.19 \mathrm{~mm} \mathrm{Hg}$ and post-CXL values were $15.77 \pm 3.14,6.37 \pm 1.39$ and $7.07 \pm 1.49 \mathrm{~mm} \mathrm{Hg}$, respectively, but the differences were not statistically significant ( $p>0.05)$ (Fig. 1).

However, when the infrared signals were analyzed, preoperative peak 1 signal amplitude increased significantly $(p<0.05)$ (Figs 2 to $3 B)$.

\section{DISCUSSION}

This study has shown that transepithelial $C X L$ treatment appears to be effective in improving $V A$ within 3 months, but statistically significant improvement was not observed in refractive outcomes in the short-term period.

Wollensak et al ${ }^{6}$ reported regression with reduction of the maximal keratometry readings by $2.01 \mathrm{D}$ after $\mathrm{CXL}$ treatment with removal of epithelium in $70 \%$ eyes with a

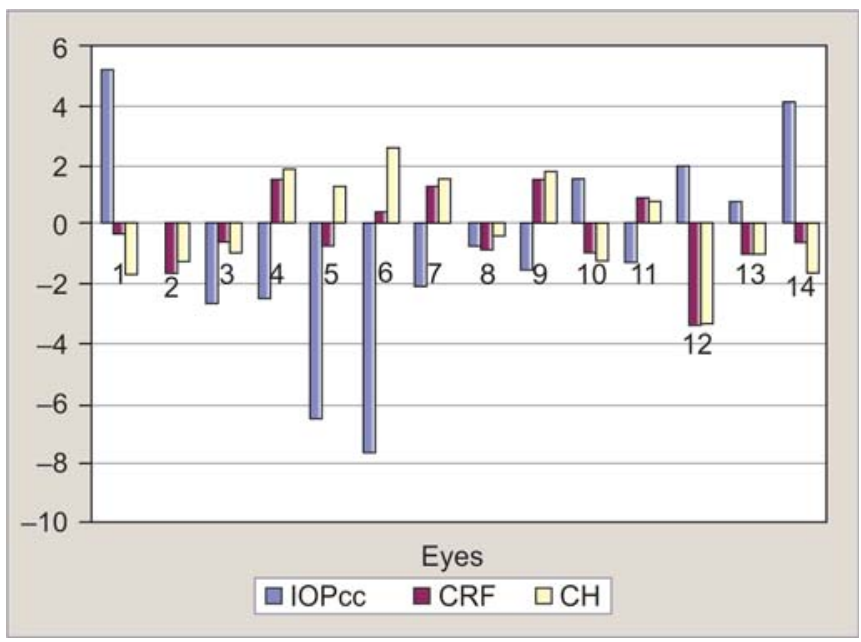

Fig. 1: Change in IOPcc, $\mathrm{CH}$ and $\mathrm{CRF}$ values after transepithelial CXL treatment

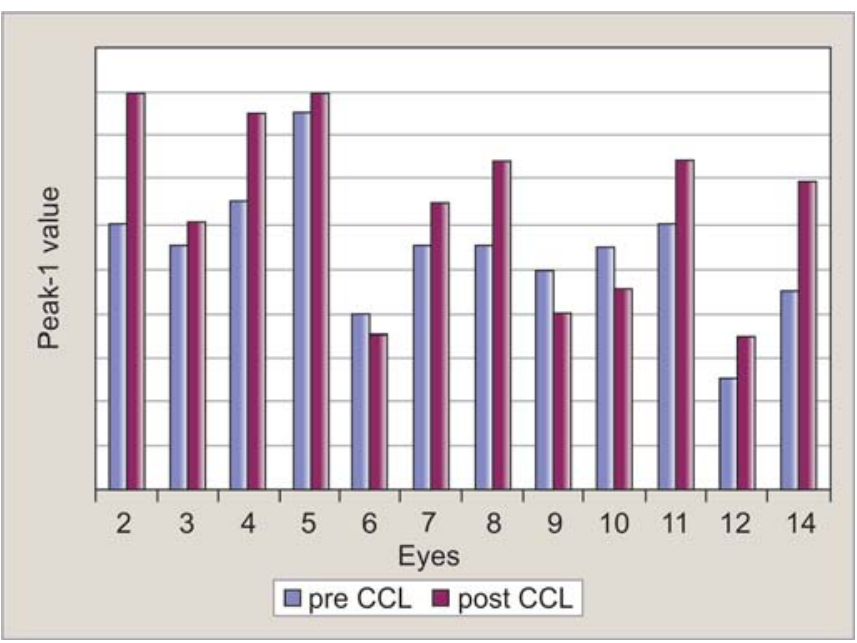

Fig. 2: Change in peak-1 value after CCL treatment

mean follow-up time of 23.2 months. In our series, we analyzed change in mean $\mathrm{K}$-value instead of maximal $\mathrm{K}$-value and improvement in mean $\mathrm{K}$-value was not significant at 3 months post-CXL. The most important advantage of transepithelial $C X L$ treatment was patient comfort after treatment and early visual recovery. In the same study, Wollensak et al ${ }^{6}$ reported an increase of

Table 1: Pre- and postoperative mean visual acuity, refractive and ORA device values after transepithelial CXL treatment

\begin{tabular}{lcrr}
\hline Parameter & Preoperative & Postoperative & $p$-value \\
\hline UCVA & $3.04 \pm 2.15$ & $4.80 \pm 2.37$ & $<0.05$ \\
BCVA & $4.98 \pm 2.48$ & $6.59 \pm 2.34$ & $<0.05$ \\
Spheric (D) & $-3.21 \pm 3.13$ & $-2.47 \pm 2.51$ & $>0.05$ \\
Cylinderic (D) & $-2.18 \pm 1.55$ & $-1.75 \pm 1.32$ & $>0.05$ \\
CCT (micron) & $464.26 \pm 27.04$ & $464.73 \pm 28.33$ & $>0.05$ \\
K-value (D) & $47.44 \pm 4.95$ & $46.33 \pm 3.62$ & $>0.05$ \\
IOPCC $(\mathrm{mm} \mathrm{Hg})$ & $16.58 \pm 4.94$ & $15.77 \pm 3.14$ & $>0.05$ \\
CRF* $(\mathrm{mm} \mathrm{Hg})$ & $6.67 \pm 1.88$ & $6.37 \pm 1.39$ & $>0.05$ \\
CH* $(\mathrm{mm} \mathrm{Hg})$ & $7.17 \pm 2.19$ & $7.07 \pm 1.49$ & $>0.05$ \\
\hline
\end{tabular}

*Included 14 eyes; BCVA: Best corrected visual acuity; UCVA: Uncorrected visual acuity; IOPcc: Intraocular pressure; CH: Corneal hysteresis; CRF : Cornea resistance factor; CCT: Central corneal thickness; K-value: Mean keratometric value 


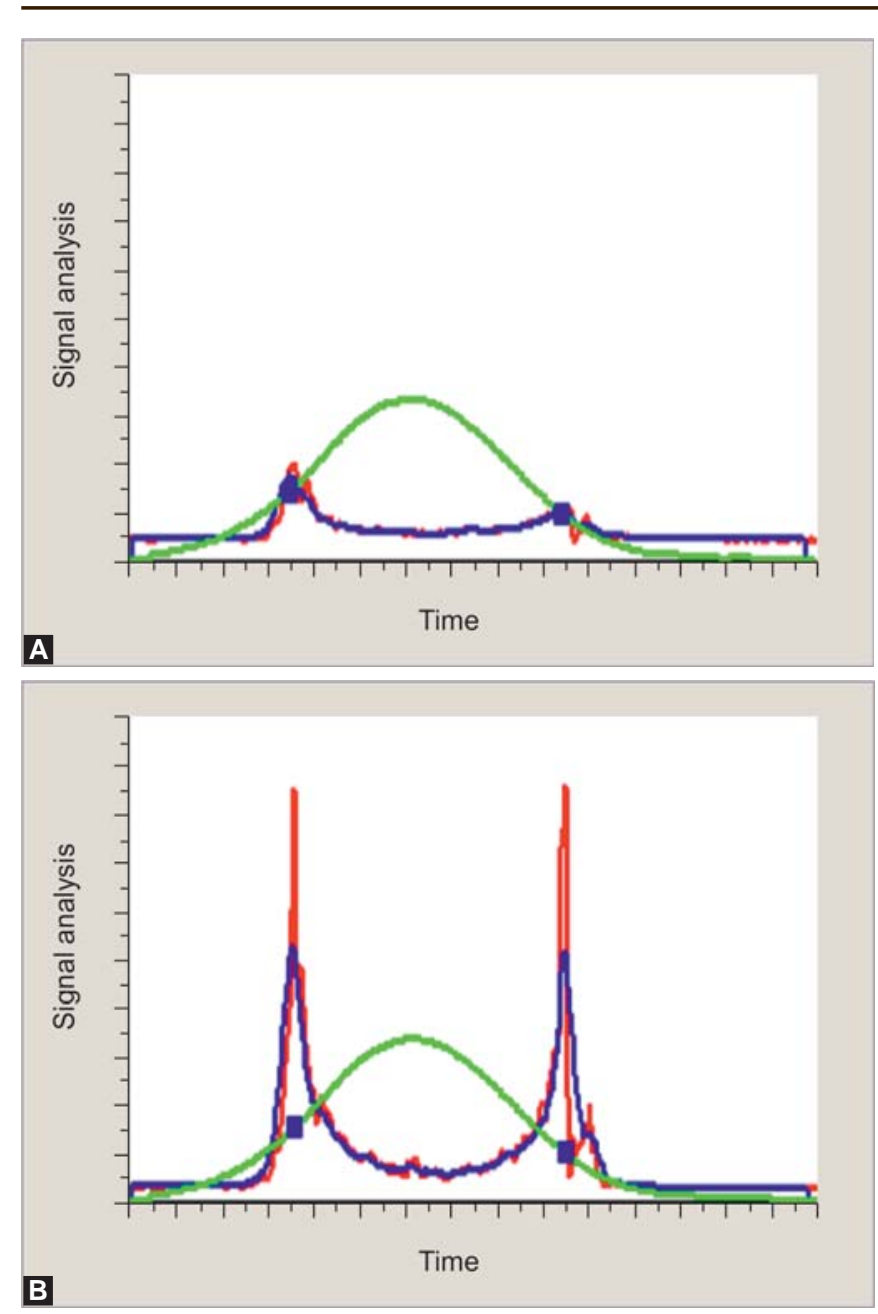

Figs $3 A$ and B: Sample case of subject who underwent $C X L$ treatment. Preoperative CRF: $7.6 \mathrm{~mm} \mathrm{Hg}, \mathrm{CH}: 6 \mathrm{~mm} \mathrm{Hg} \mathrm{(A)} \mathrm{and}$ postoperative CRF: $7 \mathrm{~mm} \mathrm{Hg}, \mathrm{CH}: 6.3 \mathrm{~mm} \mathrm{Hg}$. Postoperative peak 1 signal (red) is substantially higher (B) even though there is little change in hysteresis

$1.26 \pm 1.5$ lines in VA which is compared to $1.76 \pm 2.27$ (UCV A) and $1.61 \pm 2.52$ (BCV A) Snellen lines in our study. Sharma and Boxer Wachler presented similar results as Wollensak with transepithelial technique (Sharma M, B oxer W achler BS. Corneal collagen cross-linking with riboflavin for corneal stabilization. A A 0 meeting October 2005). This difference in visual and refractive improvement between Wollensak and the current study may be due to difference in technique (with or without epithelium removal) and follow-up periods. Pinelli et al also reported that there was no difference in $V A$ and refractive results between $C X L$ treatment with or without epithelium removal (Pinelli $\mathrm{R}$. The Italian R efractive Surgery Society (SICR). R esults using C3-R. Zurich, 2006, cross-linking meeting, oral presentation).

Increased corneal collagen fiber diameters and increased collagen stiffness have been described in diabetes mellitus and aging where collagen $C X L$ is increased. ${ }^{8-10} \mathrm{~A}$ significant increase in cornea stiffness has been measured in porcine and rabbit corneas treated by riboflavin/UVA using quantitative biomechanical stress-stain measurements. ${ }^{11}$ W ollensak et al $^{3}$ used stress-strain methodology to measure cornea stiffness after $C X L$ treatment without epithelium and they reported that collagen $C X L$ led to increase in mechanical stiffness. The dynamic bidirectional applanation process used in the Reichert ORA provides a new measure of corneal biomechanics called $\mathrm{CH}$, which is a viscoelastic parameter that is different from stiffness. Studies in the literature indicate that corneas having LA SIK, corneas with keratoconus and corneas with Fuchs' dystrophy demonstrate a general decrease in $\mathrm{CH}$ compared to corneas of normal eyes. ${ }^{12}$ In our study, we used the ORA device to measure the biomechanical effect of $C X L$ treatment. We observed insignificant changes in mean values of $\mathrm{IOPCC}, \mathrm{CH}$ and $\mathrm{CRF}$ ( $p>0.05$ ) which was similar to other studies which reported no change in $\mathrm{CRF}$ and $\mathrm{CH}$ after $\mathrm{CXL}$ treatment with removal epithelium ${ }^{14}$ ( $H$ afezi et al ORA analysis of corneal biomechanics before and after cross-linking, Zurich $\mathrm{CCL}$ meeting 2007, December).

During ORA measurement, a precisely metered air pulse is delivered to the eye, causing the cornea to move inward, past a first applanation and into a slight concavity. The infrared (IR) emitter/detector system records the IR signal during defomation under the air puff, and this signal defines two precise applanation times corresponding to two welldefined peaks in the IR signal produced by inward and outward flattening of the cornea during the applanation events. ${ }^{12}$ The amplitude of the first applantion peak in the signal, Peak 1, has been shown to correspond to corneal stiffness with a high peak 1 meaning a stiffer cornea, ${ }_{15,16}^{16}$ illustrated in Figure 4. A fter CXL treatment, we observed significantly higher peak 1 signals corresponding to an increase in corneal stiffness, similar to that reported using an epithelium-off approach. ${ }^{14}$ This value is different from $\mathrm{CH}$, which is a viscoelastic parameter and responds to changes in stiffness as well as changes in viscosity, ${ }^{17}$ both of which are altered in CXL. In the postoperative period, hysteresis was not significantly different, and yet peak 1 signals significantly increased, which show CXL treatment effectivity in terms of stiffness.

There is no report about cornea biomechanical properties after CXL treatment with intact epithelium. The concern with not removing epithelium is inadequate penetration of riboflavin, but an important point to remember is that the epithelial layer thickness is not uniform over the entire keratoconic cornea. It is thinner over the cone area, with a thicker annulus around the cone. ${ }^{18}$ In addition, the patients are receiving medicine each 2 to 3 minutes by drops which may result in damage to the epithelial junctions allowing easier penetration of riboflavin. Limited penetration may 


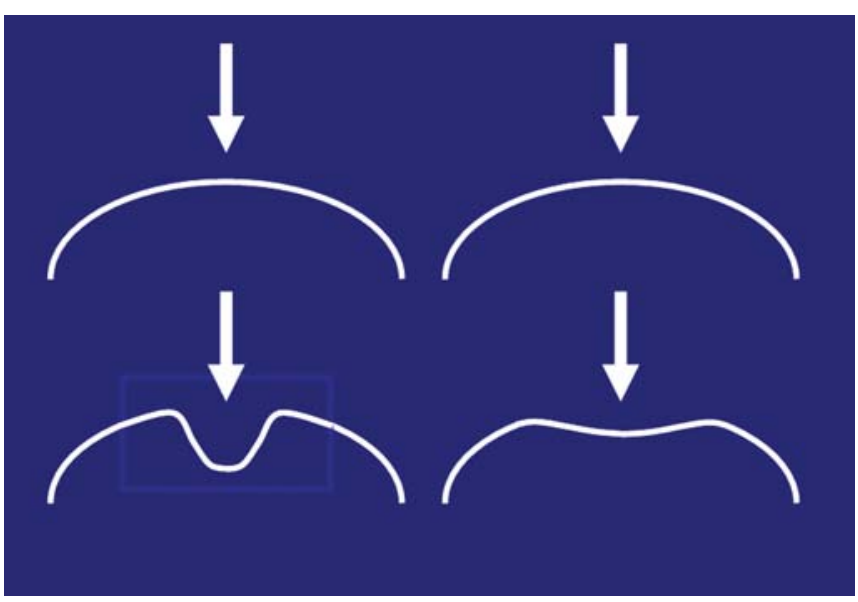

Fig. 4: Difference between soft (left) and stiff (right) cornea. The stiffer cornea has a wider area of applanation leading to a greater number of photons reflected toward the detector and a greater peak amplitude. The softer cornea has a narrower deformation and applanation area leading to fewer photons aligned with the detector and lower peak amplitudes. CXL treatment provides stiffer cornea and thus an increase in the peak magnitudes

actually be safer in terms of UVA-related damage. Podskochy et al ${ }^{19}$ showed increased keratocyte damage with UV light when the epithelium was removed. The study concluded that the epithelium may play significant role in absorbing UVA and thus protecting the cornea and deeper structures from damage.

In this study, we performed transepithelial CXL treatment and observed that there was no correlation between improvement in $\mathrm{VA}$ and refractive results. Imrovement in VA was statistically significant although changes in spheric, cylinderic and mean $\mathrm{K}$ values were not statistically significant. This significant improvement in visual acuity with nearly same refraction following a short follow-up period may be related to two-factors. The first may be a change in collagen fiber orientation with the collagen fibers becoming more regular as they are crosslinked. The second may be the correction of irregular astigmatism in the central area.

\section{CONCLUSION}

Transepithelial CXL makes the cornea stiffer as indicated by an increase in Peak 1, without changing $\mathrm{CH}$ and CRF. This is associated with a significant improvement in vision, but not refraction.

\section{REFERENCES}

1. M aurice D M. M echanics of the cornea. In: Cavanagh HD (Ed), The cornea: Transactions of the world congress on the cornea III. N ew Y ork, NY, Raven 1988;187-93.

2. Spoerl E, Huhle M, Seiler T. Induction of crosslinks in corneal tissue. Exp Eye Res 1998;66:97-103.

3. W ollensak G, Spoerl E, Seiler T. Stress-strain measurements of human and porcine cornea after riboflavin/U V A -induced crosslinking. J Cataract Refract Surg 2003;29:1780-85.
4. Tuft SJ, M oodaley LC, Gregory WM, Davidson CR, Buckley RJ. Prognostic factors to the progression of keratoconus. Ophthalmology 1994;101:439-47.

5. K ennedy RH, Bourne WM, Dyer JA. A 48-year clinical and epidemiologic study of keratoconus. Am J Ophthalmol 1986;101:267-73.

6. Wollensak G, Spoerl E, Seiler T. Riboflavin/ultraviolet-A induced collagen cross-linking for the treatment of keratoconus. A m J Ophthalmol 2003;135:620-27.

7. Chan CCK, Sharma M , W achler B SB. Effect of inferior-segment Intacs with and without C3-R on keratoconus. J Cataract R efract Surg 2007;33:75-80.

8. M alik NS, M oss SJ , A hmed N, Furth AJ, W all RS, M eek KM . A geing of the human corneal stroma: Structural and biochemical changes. Biochim Biophys A cta 1992;1138:222-28.

9. Bailey $A J$, Paul RG, Knott L. M echanism of maturation and ageing of collagen. M ech A geing Dev 1998;106:1-56.

10. Sady C, K hosrot S, Nagaraj R. A dvanced M aillard reaction and cross-linking of corneal collagen in diabetes. B iochem Biophys Res Com 1995;214:793-97.

11. Spoerl $E$, Huhle M , Seiler T. Induction of cross-links in corneal tissue. Exp Eye Res 1998;66:97-103.

12. Luce DA. Determining in vivo biomechanical properties of the cornea with an ocular response analyzer. J Cataract R efract Surg 2005;31:156-62.

13. K ohlhaas M, Spoerl E, Schilde T, Unger G, W itlig C, Pillunat $L E$. Biomechanical evidence of the distribution of cross-links in corneas treated with riboflavin and ultraviolet A light. J Cataract R efract Surg 2006 F eb;32(2):279-83.

14. Vinciguerra $P, A$ lbè $E, M$ ahmoud $A M$, Trazza $S$, Hafezi $F$, Roberts C J I Intra- and postoperative variation in ocular response analyzer parameters in keratoconic eyes after corneal crosslinking. J Refract Surg 2010A pr;28:1-8.

15. K érautret J, Colin J, Touboul D, Roberts C. Biomechanical characteristics of the ectatic cornea.J Cataract R efract Surg 2008; 34(3):510-13.

16. M ahmoud A M, Twa M D, Qazi M, Pepose J, Roberts CJ. Comparison of biomechanical and topographic parameters in normal and pathologic corneas. ARVO 2007; E-A bstract 48:1843.

17. Glass DH, Roberts CJ, Litsky AS, W eber PA. A viscoelastic biomechanical model of the cornea describing the effect of viscosity and elasticity on hysteresis. Invest Ophthalmol V is Sci 2008;49(9):3919-26.

18. Reinstein DZ, et al. E pithelial, stromal and total corneal thickness in keratoconus: Three-dimensional display with artemis veryhigh frequency digital ultrasound. J ournal of R efractive Surgery 2010;26(4):259-71.

19. Podskochy A, Gan L, Fagerholm P. A poptosis in UV -exposed rabbit corneas. Cornea 2000;19(1):99-103.

\section{ABOUT THE AUTHORS}

\section{Aylin Kılıç (Corresponding Author)}

Department of Refractive-Cataract, Dunya Eye Hospital, Istanbul Turkey, Phone: +905303136749, e-mail: aylinkilicdr@ gmail.com

\section{Cynthia J Roberts}

Professor, D epartment of O phthalmology and B iomedical Engineering The O hio State U niversity, O hio, USA 